\title{
Input-Specific Intrasynaptic Arrangements of Ionotropic Glutamate Receptors and Their Impact on Postsynaptic Responses
}

\author{
Etsuko Tarusawa, ${ }^{1,2}$ Ko Matsui, ${ }^{1,2,4}$ Timotheus Budisantoso, ${ }^{1,2}$ Elek Molnár, ${ }^{5}$ Masahiko Watanabe, ${ }^{6}$ Minoru Matsui, \\ Yugo Fukazawa, ${ }^{1,2}$ and Ryuichi Shigemoto ${ }^{1,2,3}$ \\ ${ }^{1}$ Division of Cerebral Structure, National Institute for Physiological Sciences, and ${ }^{2}$ Department of Physiological Sciences, Graduate University for Advanced \\ Studies (SOKENDAI), Okazaki 444-8787, Japan, ${ }^{3}$ SORST and ${ }^{4}$ PRESTO, Japan Science and Technology Agency, Kawaguchi 333-0012, Japan, ${ }^{5}$ Medical \\ Research Council Centre for Synaptic Plasticity, Department of Anatomy, University of Bristol, School of Medical Sciences, Bristol BS8 1TD, United \\ Kingdom, ${ }^{6}$ Department of Anatomy, Hokkaido University, School of Medicine, Sapporo 060-8638, Japan, and 7Faculty of Pharmacy, Chiba Institute of \\ Science, Choshi 288-0025, Japan
}

To examine the intrasynaptic arrangement of postsynaptic receptors in relation to the functional role of the synapse, we quantitatively analyzed the two-dimensional distribution of AMPA and NMDA receptors (AMPARs and NMDARs, respectively) using SDS-digested freeze-fracture replica labeling (SDS-FRL) and assessed the implication of distribution differences on the postsynaptic responses by simulation. In the dorsal lateral geniculate nucleus, corticogeniculate (CG) synapses were twice as large as retinogeniculate (RG) synapses but expressed similar numbers of AMPARs. Two-dimensional views of replicas revealed that AMPARs form microclusters in both synapses to a similar extent, resulting in larger AMPAR-lacking areas in the CG synapses. Despite the broad difference in the AMPAR distribution within a synapse, our simulations based on the actual receptor distributions suggested that the AMPAR quantal response at individual RG synapses is only slightly larger in amplitude, less variable, and faster in kinetics than that at CG synapses having a similar number of the receptors. NMDARs at the CG synapses were expressed twice as many as those in the RG synapses. Electrophysiological recordings confirmed a larger contribution of NMDAR relative to AMPAR-mediated responses in CG synapses. We conclude that synapse size and the density and distribution of receptors have minor influences on quantal responses and that the number of receptors acts as a predominant postsynaptic determinant of the synaptic strength mediated by both the AMPARs and NMDARs.

\section{Introduction}

Morphological characteristics of postsynaptic membrane specialization vary across synaptic contacts (Rollenhagen and Lübke, 2006), and key properties of synaptic transmission are suggested to be determined by the morphology (Franks et al., 2002, 2003; $\mathrm{Xu}-$ Friedman and Regehr, 2004). Among the characteristics, synapse size and the number and intrasynaptic distribution of receptors at individual synapses could have a large impact on the strength and variability of synaptic transmission, and therefore, receptors could be arranged in such a way to accomplish the functional role of each synapse in information processing.

\footnotetext{
Received Dec. 26, 2008; revised July 24, 2009; accepted Aug. 30, 2009.

This work was supported by grants from the SORST Japan Science and Technology Agency (JST) to R.S.; Grantin-Aid for Scientific Research on Priority Areas-Molecular Brain Sciences from the Ministry of Education, Culture, Sports, Science, and Technology of Japan (MEXT) [18022043] to Y.F.; and Grant-in-Aid for Young Scientists (A) from MEXT [18680903], Grant-in-Aid for Exploratory Research from Japan Society for the Promotion of Science [19650100], and PRESTO from JST to K.M. E.M.'s work was supported by the Medical Research Council [Grant number G0601509]. We thank Dr. M. Miyata for support and suggestions on this work, Dr. S. Itohara for forebrain-specific NR1 KO mice, and Dr. N. Kamasawa and Ms. W. Aziz for comments on this manuscript. We also thank Dr. P. Somogyi for helpful advice throughout the study.

Correspondence should be addressed to either Yugo Fukazawa or Ko Matsui (Simulation \& Electrophysiology), Division of Cerebral Structure, National Institute for Physiological Sciences, Okazaki 444-8787, Japan, E-mail: yugo@nips.ac.jp or matsui@nips.ac.jp.

D0I:10.1523/JNEUROSCI.6160-08.2009

Copyright $\odot 2009$ Society for Neuroscience $\quad$ 0270-6474/09/2912896-13\$15.00/0
}

Studies have suggested that the number of glutamate receptors expressed in different types of synapses was regulated in a connection-type dependent manner and the expression levels of the receptors were highly variable even within a single type of synapse (Nusser et al., 1998; Nyíri et al., 2003, Nicholson and Geinisman, 2009). In addition to the variability in the number of receptors, inhomogeneity of the two-dimensional distribution of AMPA receptors (AMPARs) within single postsynaptic membrane specialization has also been reported (Baude et al., 1995; Kharazia and Weinberg, 1997; Nusser et al., 1998). Our SDS-digested freeze-fracture replica immunolabeling method (SDS-FRL) (Fujimoto, 1995; Hagiwara et al., 2005; Masugi-Tokita et al., 2007) has almost one-to-one detection sensitivity to AMPARs (Tanaka et al., 2005). This method enabled us to reveal intrasynaptic microclusters of AMPARs in parallel fiber-Purkinje cell (PF-PC) synapses and their highly homogeneous distribution without such clustering in climbing fiber-Purkinje cell (CF-PC) synapses (Masugi-Tokita et al., 2007). These results suggest that the intrasynaptic distribution of AMPARs is also regulated in a connection-type dependent manner.

To explore whether different morphological characteristics at individual synapses relate to their specialized functional roles, we analyzed the functionally distinct synapses formed on the relay cells in the dorsal lateral geniculate nucleus (dLGN): retinogeniculate (RG) and corticogeniculate (CG) synapses, both of 
which are glutamatergic and express postsynaptic AMPARs and NMDA receptors (NMDARs) (Turner and Salt, 1998). Visual sensory information from the retina is received at the RG synapse, and the activation of a single optic fiber evokes postsynaptic response strong enough to reach the threshold for action potential firing, which primarily dictates the receptive field properties of the relay cells (Turner and Salt, 1998; Usrey et al., 1998). In contrast, feedback inputs from the visual cortex are received at the CG synapses and the effect of such inputs remains mostly subthreshold but does sharpen the receptive field of the relay cells (Murphy and Sillito, 1987; Sillito et al., 1993). Therefore, RG and CG synapses are functionally categorized as the driver and modulator, respectively (Sherman and Guillery, 1998).

In the present study, our quantitative SDS-FRL revealed that intrasynaptic arrangements of AMPARs and NMDARs in the two types of synapses are distinctly organized in an input- and receptor-type-specific manner. Impacts of the distinct receptor distributions on the synaptic strength and variability were assessed with electrophysiological recordings and simulations.

\section{Materials and Methods}

Animals used for morphological analysis. Male Long-Evans rats at 10 weeks of age and muscarinic acetylcholine receptor subtype 2 (M2) knockout $(\mathrm{KO})$ and wild-type mice were used. The rats and mice were raised on a $12 \mathrm{~h}$ light/dark cycle with water and food ad libitum. All animal experiments were conducted in accordance with the guidelines of the National Institute for Physiological Science's Animal Care and Use Committee.

$S D S-F R L$. The rats were anesthetized with sodium pentobarbital (50 $\mathrm{mg} / \mathrm{kg}$, i.p.) and perfused transcardially with $25 \mathrm{~mm}$ PBS for $1 \mathrm{~min}$, followed by perfusion with $2 \%$ paraformaldehyde (PFA) and $15 \%$ saturated picric acid solution in $0.1 \mathrm{M}$ phosphate buffer (PB) for $12 \mathrm{~min}$. Coronal slices (150 $\mu \mathrm{m}$ thick) were cut using a vibrating microslicer (DTK-1000; Dosaka EM) in $25 \mathrm{~mm}$ PBS. A region of the dLGN without the dorsal outer surface region containing cells receiving glutamatergic inputs from the superior colliculus (Reese, 1984) was trimmed from the slice. The trimmed slices were immersed in $30 \%$ glycerol $/ 25 \mathrm{~mm}$ PBS at $4^{\circ} \mathrm{C}$ overnight and rapidly frozen by a high pressure freezing machine (HPM010; BAL-TEC, Balzers). Frozen samples were then fractured into two parts at $-120^{\circ} \mathrm{C}$ and replicated by deposition of carbon ( $5 \mathrm{~nm}$ thick), platinum (uni-direction from $60^{\circ}, 2 \mathrm{~nm}$ ), and carbon $(20 \mathrm{~nm}$ ) in a freeze-fracture replica machine (BAF 060; BAL-TEC). After thawing, tissue debris attached to the replicas was dissolved with gentle rocking at $80^{\circ} \mathrm{C}$ for $16 \mathrm{~h}$ in a solution containing $15 \mathrm{~mm}$ Tris- $\mathrm{HCl}$ (pH8.3), 20\% sucrose, and 2.5\% SDS. The replicas were then washed in $50 \mathrm{~mm}$ Tris-buffered saline (TBS) (pH7.4) containing $0.05 \%$ bovine serum albumin (BSA) and blocked with $5 \%$ BSA in the washing buffer for $1 \mathrm{~h}$ at room temperature. The replicas were incubated with a rabbit primary antibody against GluR1-4 (panAMPAR, $3 \mu \mathrm{g} / \mathrm{ml}$ ) (the specificity of this antibody was described in ref. Nusser et al., 1998) or mouse primary antibody against NMDAR1 (NR1) (MAB363; $4 \mu \mathrm{g} / \mathrm{ml}$, Millipore) overnight at $15^{\circ} \mathrm{C}$, followed by incubation with anti-rabbit [British Biocell International (BBI)] or antimouse (GE Healthcare) secondary antibodies conjugated with $5 \mathrm{~nm}$ gold particles for $1 \mathrm{~h}$ at $37^{\circ} \mathrm{C}$. The specificity of the NR1 antibody was confirmed by labeling of replicas obtained from forebrain-specific NR1 KO mice (Iwasato et al., 2000); synaptic NR1 immunoparticles in hippocampal pyramidal cells were mostly abolished in the KO region (data not shown). Antibodies against vesicular glutamate transporter (vGluT) 2 (Miyazaki et al., 2003) and vGluT1 (Kulik et al., 2002) raised in guinea pigs were used as markers of the RG and the CG fibers (Fremeau et al., 2001; Fujiyama et al., 2003), respectively, with an anti-guinea pig secondary antibody conjugated with $10 \mathrm{~nm}$ gold particles (BBI). Although vGluTs are vesicular proteins, they are often detected on presynaptic plasma membrane and thus have been used to identify the origin of presynaptic profiles (Hagiwara et al., 2005; Masugi-Tokita et al., 2007). A rabbit antibody against M2 (AMR-002, Alomone Labs) was used, in conjunction with an anti-rabbit secondary antibody conjugated with $10 \mathrm{~nm}$ gold particles (BBI), to identify the plasma membrane of interneurons in the dLGN (Plummer et al., 1999). The speci- ficity of M2 antibody was confirmed by labeling of replicas obtained from M2 KO mice (Matsui et al., 2002); M2 immunoparticles on the P-face were all abolished in the KO tissue (data not shown). The labeled replicas were examined using a transmission electron microscope (Tecnai-10; FEI). Reliability of AMPAR localization by SDS-FRL under our fixing condition is discussed in detail in the supplemental discussions.

Quantification of immunoparticles. Images of excitatory postsynaptic specialization indicated by the presence of intramembrane particle (IMP) clusters on the exoplasmic face (E-face) (Sandri et al., 1972; Harris and Landis, 1986) accompanied by presynaptic protoplasmic face (Pface) labeled by vGluT2 or vGluT1 immunoparticles were captured at a magnification of $93,000 \times$ with a digital camera [MegaView III; Soft Imaging System (SIS)]. IMP clusters were defined as densely packed IMPs at a distance of $<15 \mathrm{~nm}$ from each other and demarcated freehand, and the areas of individual IMP clusters were measured using iTEM software (SIS). Immunoparticles within a demarcated IMP cluster and those located outside but within $30 \mathrm{~nm}$ from the edge of the IMP cluster were regarded as synaptic labeling, considering possible deviation of the immunoparticles from antigens (Matsubara et al., 1996). The total number of immunoparticles for AMPARs or NR1s in each synapse was compared with data obtained only from complete synapses. The density of the immunoparticles for AMPARs in each IMP cluster was calculated by dividing the number of the immunoparticles with the area of the IMP cluster. Analysis of the labeling densities for AMPARs was performed with data from complete and incomplete synapses since there was no significant difference in the labeling densities.

Analysis of the intrasynaptic spatial distribution of AMPAR immunoparticles. Every analysis of intrasynaptic receptor distribution was conducted only with complete synapses. The nearest neighbor distance (NND) between AMPAR immunoparticles was measured from the center of each particle to the center of the nearest immunoparticle using iTEM software. For microcluster analysis of AMPAR immunoparticles, an area of a circle with a $20 \mathrm{~nm}$ diameter was assigned to each immunoparticle, and more than three overlapping circles were defined as a microcluster. The number of microclusters detected in individual synapses was compared using simulations with the number of microclusters that occurred at random. For the random distribution analysis, 50 cases of random particle distributions in each synaptic profile were generated using a macro written in-house for the Excel software (Microsoft) (supplemental Fig. 3, available at www.jneurosci.org as supplemental material).

Measurement of postsynaptic density width from ultrathin sections. Rats were anesthetized with sodium pentobarbital $(50 \mathrm{mg} / \mathrm{kg}$, i.p. $)$ and perfused transcardially with $25 \mathrm{~mm}$ PBS, followed by perfusion with $1.6 \%$ PFA and $3 \%$ glutaraldehyde in $0.1 \mathrm{M} \mathrm{PB}(\mathrm{pH} 7.3-7.4)$ for $12 \mathrm{~min}$. Coronal slices (50 $\mu \mathrm{m}$ thick) were cut using a vibrating microslicer (VT-1000; Leica) in $0.1 \mathrm{M} \mathrm{PB}$. After washing in $0.1 \mathrm{M} \mathrm{PB}$ several times, sections were treated with $1 \% \mathrm{OsO}_{4}$ in $0.1 \mathrm{M} \mathrm{PB}$ for $1 \mathrm{~h}$, stained en bloc with $1 \%$ uranyl acetate, dehydrated with ethanol, and flat-embedded in Durcupan resin (Fluka). Serial ultrathin sections were prepared at a thickness of $70 \mathrm{~nm}$ (Ultracut S; Leica). RG and CG synapses were identified by their morphological features. Asymmetrical synapses with a large terminal containing round synaptic vesicles and pale mitochondria were classified as RG synapses, whereas asymmetrical synapses with a small terminal containing round synaptic vesicles and dark mitochondria were classified as CG synapses (Lieberman and Webster, 1974). Postsynaptic profiles containing vesicles were identified as interneurons (Rafols and Valverde, 1973) and excluded from this analysis. Serial images of identified synapses were captured from the beginning to the end of each synapse at a magnification of $93,000 \times$ with the digital camera. The postsynaptic density (PSD) width was defined by the presence of synaptic vesicles accumulated in the presynaptic active zone, postsynaptic density, and visible synaptic cleft as well as by rigid alignment of the presynaptic and postsynaptic membranes. The width of the PSD in each section was measured using iTEM software. The maximum PSD width in each synapse was used in this analysis. The scale in digital images was calibrated by a grating replica (Ted Pella).

Simulation analysis. To simulate synaptic AMPAR and NMDAR responses to quantal release of glutamate, two-dimensional distribution of immunoparticles for AMPARs or NMDARs within an IMP cluster in replica images was measured using iTEM software, and the locations of each particle were expressed in $x-y$ coordinates. Tested release sites were 
distributed at an interval of $30 \mathrm{~nm}$ within the area of each IMP cluster and the distance between the release sites and individual receptors was calculated. Instantaneous release of glutamate was assumed, and glutamate diffusion within the synaptic cleft was calculated using the analytical solution to the diffusion equation in a two-dimensional space (Uteshev and Pennefather, 1996). The concentration of glutamate was calculated assuming the size of the synaptic cleft to be $20 \mathrm{~nm}$. Such cleft width has been estimated by carefully calibrated studies and remains relatively constant in many synapses (Peters et al., 1991, Zuber et al., 2005). Moreover, a recent theoretical study has suggested that such width would be optimal for efficient synaptic transmission (Savtchenko and Rusakov, 2007). In contrast to most synaptic contacts in the CNS, RG and CG axon terminals extend well over the IMP cluster area at relay cells because these synapses are formed directly on the dendritic shaft. This is especially true for RG synapses wherein presynaptic and postsynaptic membranes are parallel to each other and the extracellular space between these two elements can be regarded essentially as a disk with no gaps or glial elements for some distance. This assumption allows the usage of the simple analytical solution rather than the more elaborate Monte-Carlo simulations (Franks et al., 2003). Time steps for AMPAR and NMDAR simulations were set as 0.5 and $5 \mu \mathrm{s}$, respectively, and the glutamate concentration profile at each receptor location was calculated using the Excel software. Unless otherwise noted, the number of glutamate molecules released $(N)$ was set at 2000 molecules, and the diffusion coefficient of glutamate $(D)$ was set at $0.3 \mu \mathrm{m}^{2} / \mathrm{ms}$ (Barbour, 2001; Rusakov, 2001; Franks et al., 2003, Nielsen et al., 2004, Zheng et al., 2008). Validity of the selection of these parameters is discussed in detail in the supplemental discussion. In short, our main conclusion was not affected by the selection of parameters within the range tested $(1000<N<5000,0.1<D<0.76$; supplemental Fig. 4, available at www.jneurosci.org as supplemental material). An AMPAR kinetic model with rates determined at $32-35^{\circ} \mathrm{C}$ by Wadiche and Jahr (2001) was run against the glutamate transients to calculate the Pos of individual AMPARs using AxoGraph X software (AxoGraph Scientific). The number of AMPARs opened by a single vesicular release at a given release site was calculated by summing all the Pos of AMPARs within a synapse. Spatial maps of the normalized peak AMPAR responses were illustrated in gray scale using Origin software (OriginLab). The rise time (10-90\%) and decay time (time from the peak to $36.8 \%$ of the peak) were calculated using AxoGraph X and Excel software. For simulation of AMPAR-mediated responses in the lattice distribution, an identical number of immunoparticles detected in a synapse by replica labeling was rearranged within the same synaptic area using Excel software. For simulation of NMDAR-mediated responses, an NMDAR kinetic model [Long-pulse L-glutamate model described in the study by Lester and Jahr (1992)] was modified to simulate responses at $35^{\circ} \mathrm{C}$ by scaling all the rate constants by the NMDAR $Q_{10}$ of 2.5 (McLarnon and Curry, 1990; Chen et al., 2001). Blockade of NMDARs by $\mathrm{Mg}^{2+}$ was not considered in the modeling.

Electrophysiology. Brain slices containing dLGN were prepared from young Long-Evans rats (P21 to P30). The animals were anesthetized by inhalation of halothane before decapitation, and the brain was sliced in ice-cold solution containing (in $\mathrm{mm}$ ) $119.0 \mathrm{NaCl}, 2.5 \mathrm{KCl}, 0.1 \mathrm{CaCl}_{2}, 3.2$ $\mathrm{MgCl}_{2}, 1.0 \mathrm{NaH}_{2} \mathrm{PO}_{4}, 26.2 \mathrm{NaHCO}_{3}$, and 11.0 glucose (saturated with $95 \% \mathrm{O}_{2}-5 \% \mathrm{CO}_{2}$ ). Following the procedure by Turner and Salt (1998), the brain was glued at an angle so that the sensory and cortical inputs could be preserved in the sliced tissue. Slices were cut at $300-350 \mu \mathrm{m}$ sections using a microslicer (PRO7; Dosaka EM). The slices were then incubated in the above solution with $\mathrm{CaCl}_{2}$ and $\mathrm{MgCl}_{2}$ concentrations substituted to $2.0 \mathrm{~mm}$ and $1.3 \mathrm{~mm}$, respectively, at $34^{\circ} \mathrm{C}$ for $30 \mathrm{~min}$ and then stored at room temperature. During the recording, slices were superfused with the latter solution at room temperature with the addition of $100 \mu \mathrm{M}$ picrotoxin to block $\mathrm{GABA}_{\mathrm{A}}$ receptors. Whole-cell voltageclamp recordings were made under visual guidance $(40 \times$ or $60 \times$ waterimmersion objective, Olympus upright microscope BX51WI equipped with infrared-DIC) using Axopatch 200B patch-clamp amplifier (MDS Analytical Technologies). Signals were filtered at $2 \mathrm{kHz}$ and digitized at $10 \mathrm{kHz}$ with Digidata 1322A using pClamp 8 acquisition software (MDS Analytical Technologies). Patch electrodes with resistances of 3-4 M $\Omega$ were used with the internal solution (in $\mathrm{mm}$ ): $35 \mathrm{CsF}, 100 \mathrm{CsCl}, 10$ HEPES, and 10 EGTA (pH 7.2 with $\mathrm{CsOH}$ titration) with the addition of
$100 \mu \mathrm{M}$ calcein. Holding potentials were corrected for the liquid junction potential of $7 \mathrm{mV}$. After each recording, only the recordings from the relay cells were selected by visual inspection of the cell morphology using epifluorescence microscopy. The relay cells were distinguished from the local inhibitory interneurons by their larger sizes and by the presence of three or more dendrites coming off from the cell bodies. RG and CG fiber stimulations were accomplished by placing a bipolar stimulating electrode near the caudal border of the optic tract and near the nucleus reticularis thalami region, respectively. Evoked EPSCs from each fiber were readily distinguishable as the RG fiber input showed step-like increases in the amplitude with increasing magnitude of stimuli with each step corresponding to recruitment of single RG fiber activations. Stimulus intensity was minimized for the RG fiber stimulation to accomplish putative single fiber stimulation. Increase in the CG fiber input with increasing stimulus magnitude was more graded suggesting involvement of multiple CG fiber input, each with a small number of release sites. In addition, marked paired-pulse depression was observed for RG fiber input whereas CG fiber input showed paired-pulse facilitation as demonstrated in Figure 8. To evoke asynchronous EPSCs, extracellular divalent salts were substituted with $1 \mathrm{mM} \mathrm{SrCl}_{2}$ and 2 $\mathrm{mm} \mathrm{MgCl} 2$ or with $3.3 \mathrm{mM} \mathrm{SrCl}_{2}$, and $100 \mu \mathrm{M}$ DL-AP5 was supplemented to the extracellular solution to block the possible contamination of the NMDA component of the EPSCs (supplemental Fig. 6, available at www.jneurosci.org as supplemental material). Asynchronous quantal response detection and other electrophysiological data analysis were performed with AxoGraph X.

Statistical analysis. Statistical analysis was conducted by SPSS. The mean values between groups were compared by Student's $t$ test and median values between groups were analyzed by Mann-Whitney $U$ test and Wilcoxon signed-rank test. Normality and correlation was examined by Shapiro-Wilk's $W$ test and Spearman's rank-order correlation test, respectively. Numbers shown in parentheses indicate mean \pm SD or median values.

\section{Results}

\section{Replica images of RG and CG synapses were identified by vGluT2 and vGluT1 labelings in the rat dLGN}

The postsynaptic membrane specialization of glutamatergic synapses in a freeze-fracture replica is indicated by a cluster of IMPs on the E-face of the plasma membrane (Sandri et al., 1972; Harris and Landis, 1986), and is often accompanied by the P-face of its presynaptic plasma membrane. In the dLGN, such IMP clusters were often observed in putative dendritic shafts, and they were always immunopositive for mouse primary antibodies against NR1, suggesting that these IMP clusters represent the postsynaptic specialization of glutamatergic synapses (Figs. 1, 7; supplemental Fig. 2, supplemental Table 4, available at www.jneurosci. org as supplemental material). These IMP clusters could be approximately categorized into two types: one often found as multiple IMP clusters on a large postsynaptic E-face (Fig. $1 A, B$ ) and the other as a single IMP cluster on a slender postsynaptic E-face (Fig. 1C). In many cases, the former was accompanied by a large presynaptic P-face (Fig. $1 A$, shown in blue), whereas the latter by a small presynaptic P-face (Fig. $1 C$, shown in blue). The two distinct features correspond to the ultrastructural characteristics of RG and CG synapses, respectively (Erişir et al., 1997; Van Horn et al., 2000; Li et al., 2003). To confirm the identity of these synapses on the replica, we labeled for vGluT 2 and 1, which are selectively expressed in RG and CG fibers in the dLGN, respectively (see Materials and Methods) (Fremeau et al., 2001; Fujiyama et al., 2003). In accordance with their morphological features, the large presynaptic P-faces were always labeled for vGluT2 (Fig. 1 $A, B$ ) while labeling for vGluT1 was exclusively found in the small presynaptic P-face (Fig. $1 C$ ), indicating that the former was derived from the RG fiber and the latter from the CG fiber. Since RG fibers also innervate interneurons in the dLGN, we further identified the RG-interneuron (RG-IN) synapses by labeling for M2, which is selectively expressed in interneurons in the dLGN (Plummer et al., 1999), and analyzed the 


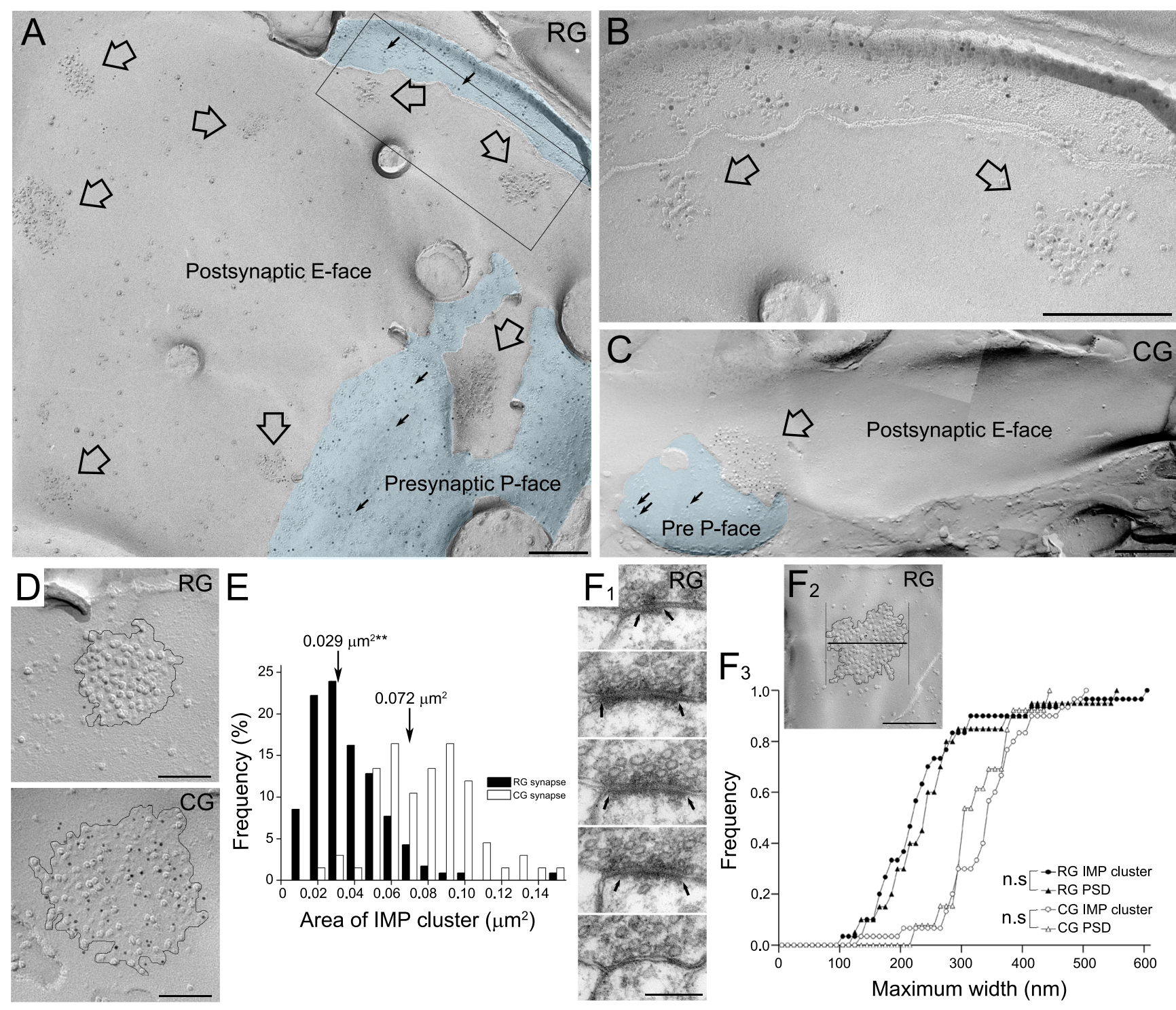

Figure 1. The synaptic areas of RG synapses are smaller than those of (G synapses in the rat dLGN. Replicas obtained from the dLGN were labeled with immunoparticles for vGluT2 (A) or vGluT1 (C) and for NR1. The P-faces labeled with immunoparticles for vGluT2 or vGluT1 are illustrated as blue areas. Immunoparticles for NR1 (5 nm) were found on IMP clusters on the E-face (large arrows), indicating that they are glutamatergic postsynaptic specialization. $A$, Multiple IMP clusters on a wide E-face accompanied by large P-faces (blue areas) labeled with immunoparticles for vGluT2 (10 $\mathrm{nm}$, small arrows) were identified as RG synapses. $\boldsymbol{B}$, A magnified image of IMP clusters (large arrows) in a rectangular area in $\boldsymbol{A}$. $\boldsymbol{C}$, Single IMP cluster on a slender E-face accompanied by a small P-face labeled with immunoparticles for vGluT1 (10 nm, small arrows) was identified as a CG synapse. Scale bar, $250 \mathrm{~nm}$. $\boldsymbol{D}$, Representative IMP clusters of RG $(\boldsymbol{A})$ and CG (B) synapse labeled with NR1 immunoparticles $(5 \mathrm{~nm})$. Black lines indicate boundaries of IMP clusters. Scale bar, $100 \mathrm{~nm}$. $E$, The distribution of IMP cluster areas from CG, but not RG, synapses followed Gaussian distribution (RG synapse: $p<0.01, n=117$ synapses; $(G$ synapse: $p=0.472, n=67$ synapses; Shapiro-Wilk's $W$ test). Arrows indicate median values for RG and (G synapses, which are significantly different $\left({ }^{* *} p<0.01\right.$, Mann-Whitney $U$ test). $\boldsymbol{F}_{1}-\boldsymbol{F}_{3}$, Comparison of the maximum PSD widths and IMP cluster widths in the RG and the $\left(G\right.$ synapses. $\boldsymbol{F}_{1}$, Serial ultrathin sections of a RG synapse. Arrows indicate the edge of PSD. $\boldsymbol{F}_{2}$, A thick line with double arrow indicates the maximum width of an IMP cluster. $\boldsymbol{F}_{3}$, Cumulative frequency plots of maximum widths of the PSDs and IMP clusters. The maximum widths of the PSD and IMP cluster were not significantly different (RG: $p=0.476$, G $: p=0.425$, Mann-Whitney $U$ test). Scale bar in $\boldsymbol{F}_{1}$ and $\boldsymbol{F}_{2}, 200 \mathrm{~nm}$.

morphological characteristics and receptor distributions in the RG-IN synapses separately (supplemental Figs. 1, 2, available at www.jneurosci.org as supplemental material). Less than $15 \%$ of the RG synapses identified by vGluT2 labeling were colabeled for M2, thereby considered to be RG-IN synapses (supplemental Tables 2, 4, available at www.jneurosci.org as supplemental material).

The surface area of the RG synapses is smaller than that of the CG synapses

To compare the size of the synapses formed by RG and CG on relay cells, we measured the surface areas of the IMP clusters that were replicated entirely (complete IMP clusters, Fig. $1 D$ ) in five rats. The distribution of the areas of the two types of synapses overlapped (Fig. $1 E$ ), but the RG synapses (median $=0.029 \mu \mathrm{m}^{2}$, $n=117$ synapses) were significantly smaller (Mann-Whitney $U$ test, $p<0.001$ ) than the CG synapses (median $=0.072 \mu \mathrm{m}^{2}, n=$ 67 synapses). To verify whether the area of the IMP cluster in the replicas was comparable to that of PSD visualized in conventional ultrathin sections, we also analyzed the maximum PSD widths of RG and CG synapses in serial ultrathin sections (Fig. $1 F_{1}, F_{3}$, triangles). The maximum PSD width of the RG synapses (median $=245.7 \mathrm{~nm}, n=20$ synapses) was significantly shorter ( $p=$ 0.003 , Mann-Whitney $U$ test) than that of the CG synapses (median $=301.9 \mathrm{~nm}, n=13$ synapses). The maximum horizontal width of the IMP clusters (Fig. $1 F_{2}, F_{3}$, circles) was not significantly different from the maximum PSD width in both types of 

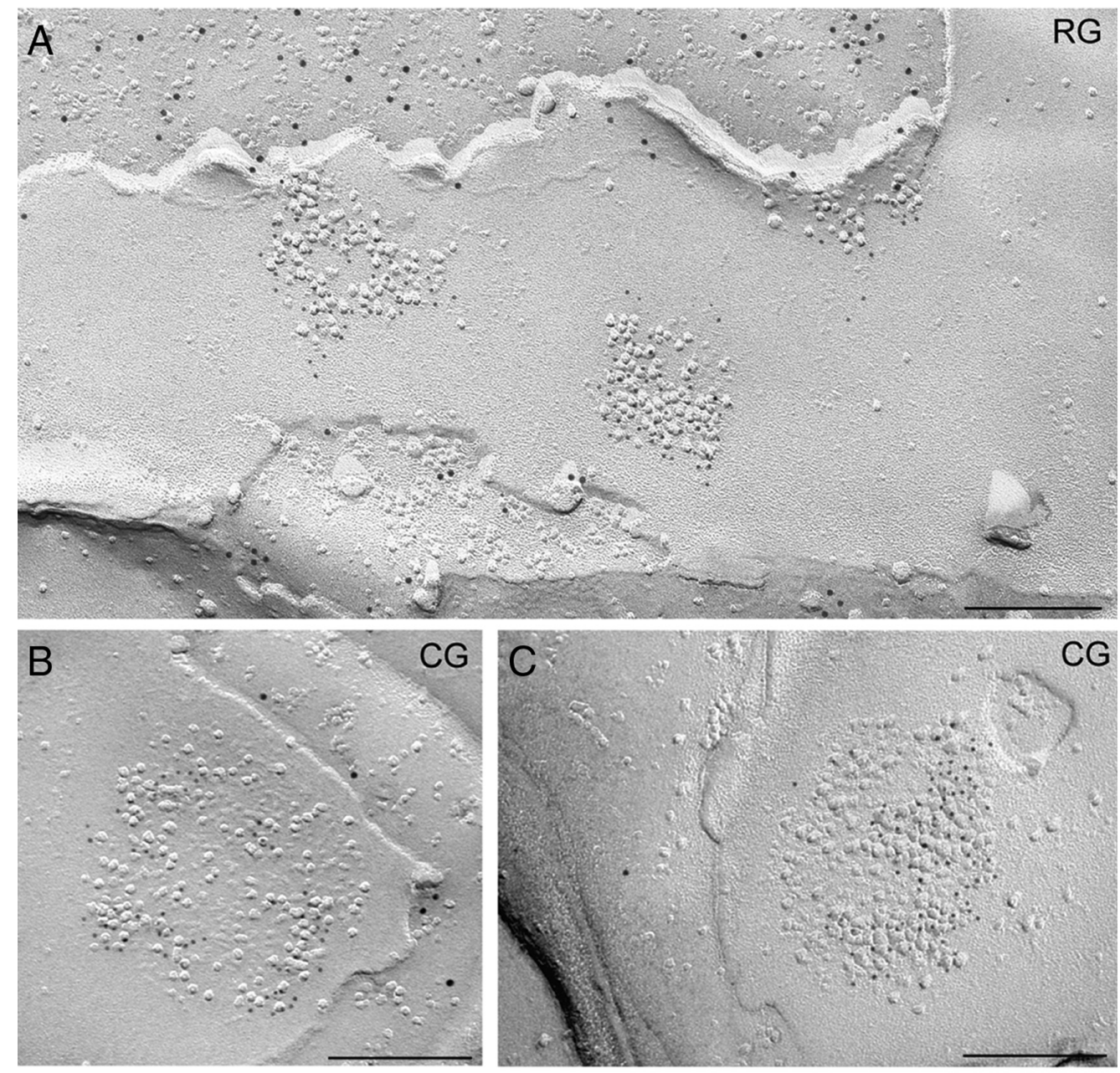

D
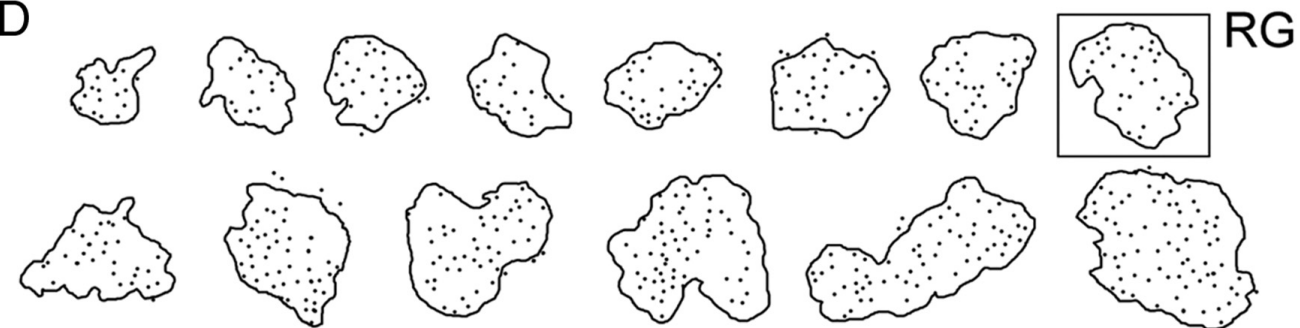

$E$
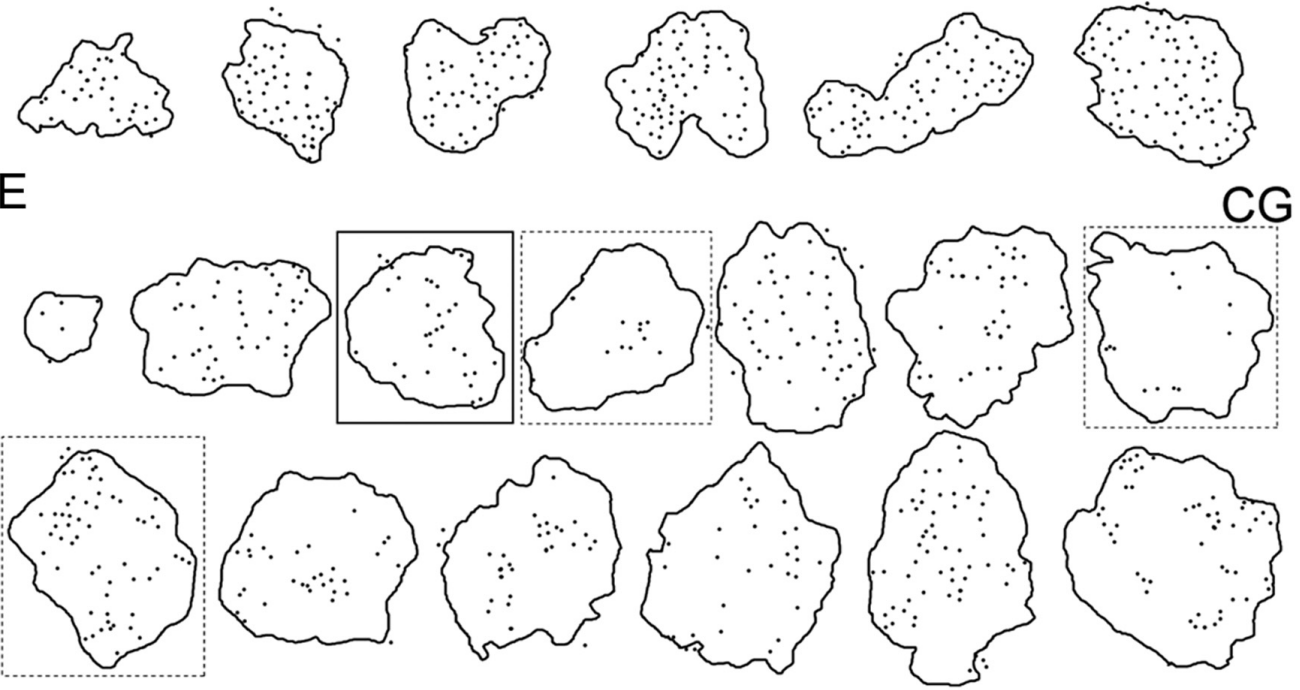

Figure 2. Differential two-dimensional distribution of AMPAR immunoparticles in $\mathrm{RG}$ and $C G$ synapses. $A, R G$ synapses were identified by vGluT2 immunoparticles (10 $\mathrm{nm})$ on the accompanied P-face of presynaptic terminal. Immunoparticles for AMPARs $(5 \mathrm{~nm})$ entirely covered the IMP cluster. B, C, CG synapses were identified by vGluT1 immunoparticles (10 nm) on the accompanied P-face of presynaptic terminal. Immunoparticles for AMPARs $(5 \mathrm{~nm})$ were distributed as multiple microclusters within the IMP cluster area. Note that immunoparticles for AMPARs are found over clearly visible IMPs in both RG and CG synapses. Scale bar, $200 \mathrm{~nm}$. D, E, Some example distributions of the AMPAR immunoparticle in individual synapses obtained from rat \#1 are shown. The AMPAR immunoparticles distribute almost entirely over the IMP clusters in RG synapses $(\boldsymbol{D})$ but not in $(G$ synapses $(\boldsymbol{E})$ with similar local densities of the immunoparticles between the two synapses. Profiles boxed with a solid line and a broken line were used for simulation of release location-dependent AMPAR responses presented in Figure 5 and supplemental Figure 5, available at www.jneurosci.org as supplemental material, respectively. 

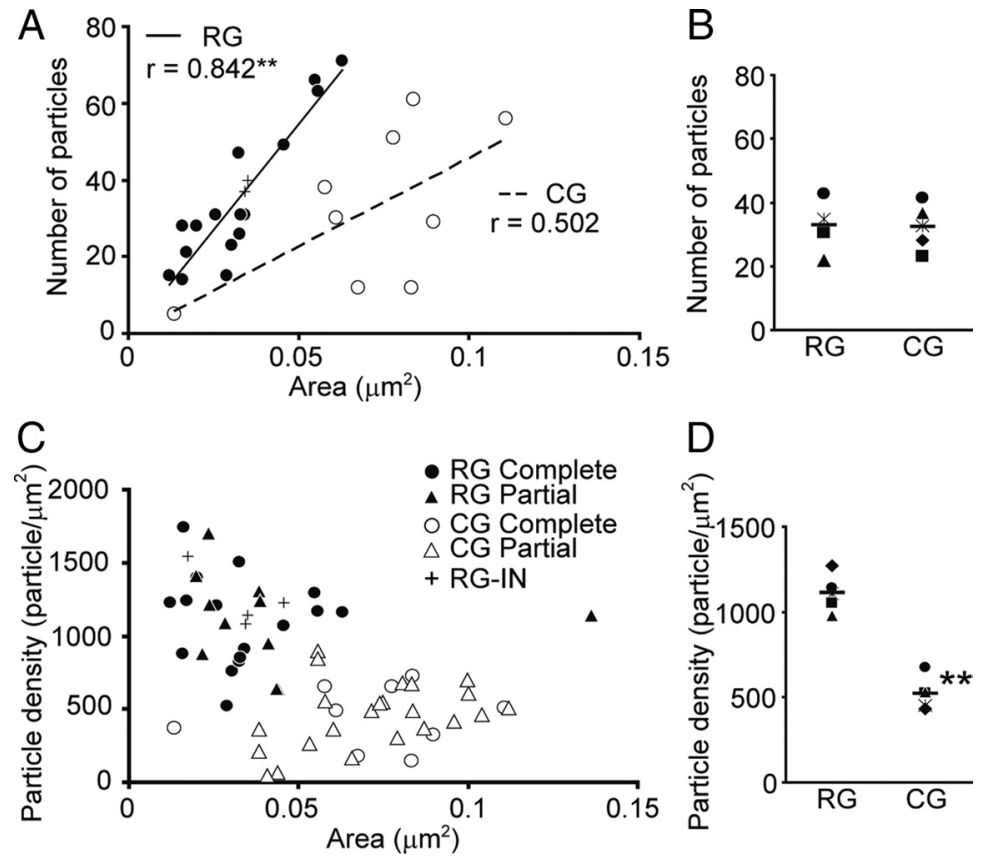

Figure 3. $R G$ and $C G$ synapses express similar numbers of AMPARs with different average densities. Areas of IMP clusters and numbers of AMPAR immunoparticles were measured in replica images. Data obtained from complete $(\boldsymbol{A})$ and complete and incomplete (C) IMP clusters in rat \#4 are shown, and the average values from five rats are plotted in $(\boldsymbol{B})$ and $(\boldsymbol{D})$. $\boldsymbol{A}$, The synaptic area and the number of AMPAR immunoparticles in RG synapses (O) showed higher positive correlation than in CG synapses $(O)(r=$ coefficient of correlation, ${ }^{* *} p<0.01$, Spearman's rank-order test). $\boldsymbol{A}, \boldsymbol{C}, \mathrm{RG}$-interneuron (RG-IN) synapses are shown with $(+)$. $\boldsymbol{B}, \mathrm{RG}$ and CG synapses showed similar average numbers of AMPAR immunoparticles per synapse ( $p=0.872$, Student's $t$ test, $n=$ 5 rats). C, $D, R G$ synapses showed twice higher density of AMPAR immunoparticles than CG synapses ( ${ }^{* *} p<0.01$, Student's $t$ test, $n=5$ rats). The mean values of each rat are indicated with the same symbols for both RG and CG synapses, and values of five rats are indicated with horizontal bars in $\boldsymbol{B}$ and $\boldsymbol{D}$.

synapses (maximum width of the IMP cluster: for the RG synapse, median $=224.6 \mathrm{~nm}, n=30$ synapses, and $p=0.476$; for the CG synapse, median $=347.31 \mathrm{~nm}, n=30$ synapses, and $p=$ 0.425 , Mann-Whitney $U$ test). These results indicate that the IMP clusters on the E-face correspond to PSDs and that, on average, CG synapses are twice as large as RG synapses.

\section{AMPARs are differentially distributed in the RG and CG synapses}

We labeled AMPARs with an antibody that specifically recognizes all four subunits (GluR1-4) of the AMPARs (Nusser et al., 1998; Tanaka et al., 2005; Masugi-Tokita et al., 2007). Immunoparticles for AMPARs in the RG synapses were found all over the IMP cluster (Fig. 2A,D; supplemental Fig. 2, available at www.jneurosci.org as supplemental material). In the CG synapses, AMPAR immunoparticles were often found only in certain parts of the IMP clusters. These aggregates of AMPAR immunoparticles appeared randomly over individual synapses (Fig. $2 B, C, E$ ). Such uneven distribution of AMPAR immunoparticles was a consistent feature of the CG synapses and did not depend on the size or the shape of the IMP cluster (Fig. 2E). Interestingly, these aggregates coincided with clearly represented large IMPs $(\sim 14 \mathrm{~nm}$ in diameter, Figs. $2 B, C)$. The number of immunoparticles for AMPARs in individual synapses was positively correlated with the synaptic area (Fig. 3A; supplemental Table 2 , available at www.jneurosci.org as supplemental material) both in the RG ( $y=963 x+2.9, n=103, p<0.001$, Spearman's rank-order test) and CG synapses ( $y=474 x+3.4, n=62, p<0.001$, Spearman's rank-order test), and the correlation coefficient value was significantly higher in the RG synapses $(r=0.83 \pm 0.031$ in the RG synapses and $r=0.58 \pm 0.074$ in the CG synapse; $n=5$ rats; $p<$
0.001 , Student's $t$ test). The mean number of immunoparticles per synapse was similar between the RG and CG synapses ( $33 \pm 7.7$ in the RG synapses and $32 \pm 7.1$ in the $\mathrm{CG}$ synapses; $n=5$ rats; $p=0.872$, Student's $t$ test) (Fig. 3B; supplemental Table 2, available at www.jneurosci.org as supplemental material). The density of the immunoparticles showed no significant correlation with the synaptic area in the RG and CG synapses (Fig. 3C; supplemental Table 2, available at www.jneurosci.org as supplemental material). Because of the distinct synaptic areas of the RG and CG synapses, the average density of immunoparticles in the RG synapses was more than twice as high as that in the CG synapses $\left(1112 \pm 108 / \mu \mathrm{m}^{2}\right.$ in the RG synapses and $523 \pm 94.9 / \mu \mathrm{m}^{2}$ in the CG synapses; $n=5$ rats; $p<0.001$, Student's $t$ test) (Fig. 3D; supplemental Table 2, available at www.jneurosci.org as supplemental material). Coefficient of variance (CV) of particle density in the RG synapses was significantly smaller than that in the CG synapses $(0.3 \pm 0.08$ in the RG synapses and $0.5 \pm 0.10$ in the CG synapses; $n=5$ rats; $p=0.008$, Student's $t$ test; supplemental Table 2, available at www.jneurosci.org as supplemental material).

Despite the difference in the average particle density between RG and CG synapses, we noticed that the local density of particles within these synapses appeared similar (Fig. $2 D, E)$. To quantitatively assess the intrasynaptic spatial arrangements of AMPARs, we analyzed the nearest neighbor distances (NNDs) of the AMPAR immunoparticles in three rats (Fig. 4A; supplemental Table 3, available at www.jneurosci.org as supplemental material). There was no significant difference in the distribution of NNDs between the RG and CG synapses of all animals (Fig. 4A, supplemental Table 3, available at www.jneurosci.org as supplemental material), and $>85 \%$ of the immunoparticles were located within a distance of $40 \mathrm{~nm}$ from each other, suggesting that the local density of AMPARs was similar in both types of synapses. To further characterize the arrangement of these local clusters of AMPARs in the RG and CG synapses, we defined "microclusters" as more than three AMPAR particles located within $20 \mathrm{~nm}$ ( the median value of NNDs) (Fig. $4 A$ ) from each other as an arbitrary criterion for the detection of local clusters (Fig. $4 B, C$, white circles). The number of microclusters per synapse was strongly correlated with the number of AMPAR immunoparticles in both RG and CG synapses with similar clustering rates (Fig. $4 D$, regression line: $y=$ $0.10 x+0.20$ in the RG synapses and $y=0.12 x-1.03$ in the CG synapses). To determine whether microclusters of receptor are formed by active processes or whether such clustering can be expected from just stochastic distribution of receptors, we compared the number of microclusters detected in the actual synapses with the number of microclusters coincidentally formed when the same particles were randomly distributed within the same synaptic profile (Fig. $4 E$, supplemental Fig. 3, available at www.jneurosci.org as supplemental material). The number of microclusters was significantly smaller when the particles were randomly distributed in the simulated CG synapses (7 of 9). In the RG synapses, the number of microclusters in 
the simulated random distributions was significantly smaller in 9 of 16 synapses. Thus, clustering of AMPARs appears to be actively regulated in the real RG and CG synapses. These results demonstrated that AMPARs exist in the clustered forms in both types of synapses and their clustering patterns in relation to the expression level of AMPARs are similar between the two types of synapses, leaving larger AMPAR-lacking areas (open spaces) in the CG synapses.

Effect of microclusters, open spaces, and release locations on the AMPARmediated synaptic quantal responses As demonstrated in Figure 4, AMPARs are unevenly distributed over the postsynaptic membrane specialization particularly in the CG synapses having both microclusters and open spaces devoid of apparent AMPAR expression. As morphological characteristics including receptor distributions have been suggested to influence synaptic property (Franks et al., 2002, 2003; Xu-Friedman and Regehr, 2004), it appeared conceivable that vesicular release of glutamate in close proximity to AMPAR microclusters would result in larger synaptic responses than glutamate release at AMPAR-lacking open spaces. If the release randomly occurs over the region of synaptic contact, AMPAR-mediated synaptic responses in CG synapses would be more variable and have smaller amplitudes on average than those in RG synapses. To address this, we simulated glutamate diffusion within the synaptic cleft and calculated the glutamate concentration transient experienced by individual AMPARs located by the replica labeling. The open probabilities (Pos) of each AMPAR were calculated using the kinetic model of AMPARs (Wadiche and Jahr, 2001; Matsui et al., 2005), and the cell's total response to a vesicular release of glutamate was calculated by summation of the Pos of all AMPARs within the synapse (Fig. 5).

We first considered situations caused by the release of glutamate at two distinct locations within a CG synapse: one at an "ON cluster" release site and the other at an "OFF cluster" release site (Figs. 5A, B; supplemental Movie 1, available at www.jneurosci. org as supplemental material). In Figure $5 B$, glutamate transients at each AMPAR location (upper traces) and the responses of each receptor to such glutamate transients (lower traces) have been plotted. ON cluster release resulted in more AMPARs exposed to high concentrations of glutamate and thus resulted in receptors with higher Pos than OFF cluster release. The sums of all Pos (open AMPAR number) for ON cluster and OFF cluster releases are plotted as red and yellow traces in Figure $5 C$, respectively. Contrary to our initial intuition, the peak of the open AMPAR number resulting from the $\mathrm{ON}$ cluster release was only slightly higher than that from the OFF cluster release (Fig. 5C; a difference of 1.5 open AMPARs at the peak). Closer inspection of the responses of individual receptors revealed that although ON cluster release causes larger responses in a subset of AMPARs, due to the presence of numerous receptors that respond moderately, the difference in the total response does not deviate much from that of the OFF cluster release (Fig. 5B, supplemental Movie 1, avail-
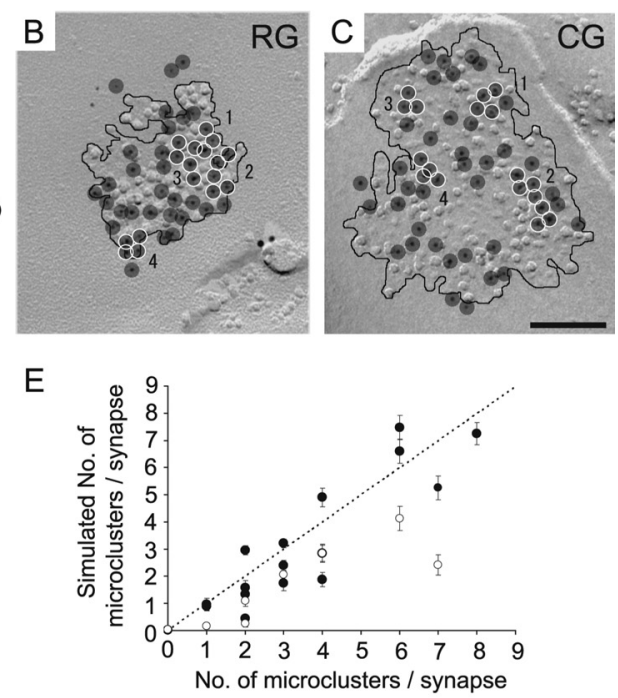

o CG synapse

edian values

$20.4 \mathrm{~nm}$ (Syn. $n=16$, NND $n=558)$ : $21.2 \mathrm{~nm}($ Syn. $n=9, \mathrm{NND} n=286$ ) ( 10 -test)

NND (nm)

$100 \quad 120$

Clustering patterns of synaptic AMPARs are identical between RG and CG synapses. $\boldsymbol{A}$, The NNDs for each AMPAR immunoARs (microclusters), an arbitrary circle of $20 \mathrm{~nm}$ diameter was given to individual immunoparticles in original replica images ( $\boldsymbol{B}$ and $\boldsymbol{C}$

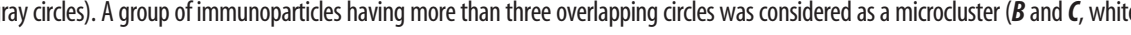
lower than those found in most of the CG synapses or those in more than half of the RG synapses. Black lines in $\boldsymbol{B}$ and $\boldsymbol{C}$ indicate the edge of IMP cluster. Scale bar, $100 \mathrm{~nm}$. Error bars indicate $95 \%$ confident interval of the mean.

able at www.jneurosci.org as supplemental material). The peaks of the open AMPAR number induced by release at various locations within a synapse are expressed in shades of gray, as shown in Figure $5 D$. Although there was a tendency for the open AMPAR number to be higher for release closer to receptor microclusters, release in open spaces produced sizable responses (Figs. 5C,D; supplemental Fig. 5, available at www.jneurosci.org as supplemental material). Similar analysis was conducted for RG synapses, as shown in Figure 5E. The peaks of the open AMPAR number in this RG synapse, which had a similar number of AMPAR immunoparticles as the CG synapse example, were slightly larger. This difference can be explained by the lack of open spaces and/or the smaller synaptic area in the RG synapses, concentrating AMPARs close to the postulated release sites.

To further evaluate whether the presence of AMPAR microclusters and open spaces has any significance to synaptic transmission on average, we considered a hypothetical situation where AMPARs were scattered evenly throughout the synaptic area in a lattice distribution (see Materials and Methods, Fig. 5G). Using the same synaptic area and receptor number as the CG synapse example shown in Figure 5A, AMPAR responses were calculated (Fig. 5G). We found that the average number of open AMPARs was nearly the same but the CV value of open AMPARs was slightly lowered in the lattice distribution [real (clustered) distribution, mean $=4.21, \mathrm{CV}=0.14$; lattice distribution; mean $=4.24, \mathrm{CV}=0.12$ ]. Similar comparison was made on all RG and CG synapses from rat \#4 (Fig. $5 H, I$ ) and the effect of the real (clustered) distribution on the $\mathrm{CV}$ was found to be significant only in the CG synapses (Fig. $5 I$, average CV $=0.17$ in real and 0.13 in lattice distribution, $p=0.043$, Wilcoxon signed-rank test). This indicates that the open spaces of AMPAR distribution have no impact on the average synaptic strength but could affect the 


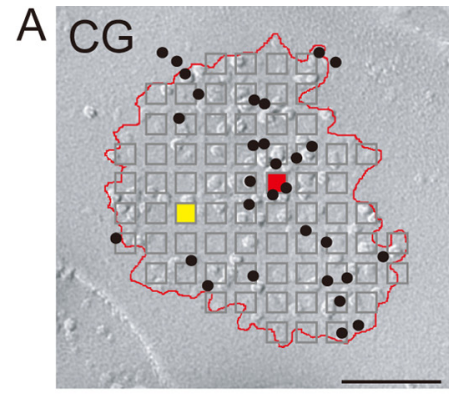

C

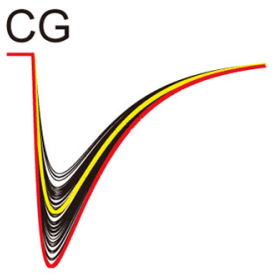

E

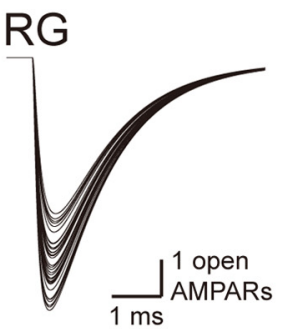

G

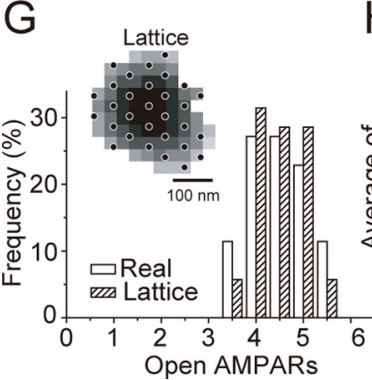

$\mathrm{H}$
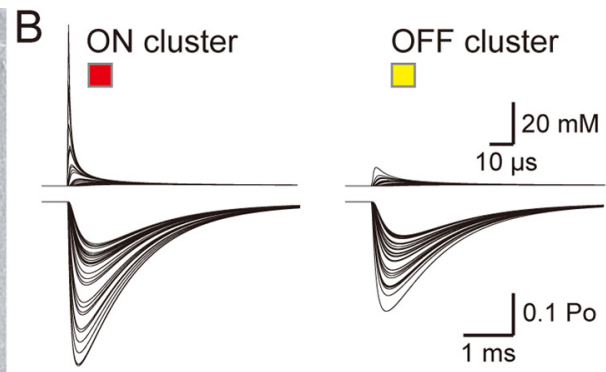

D

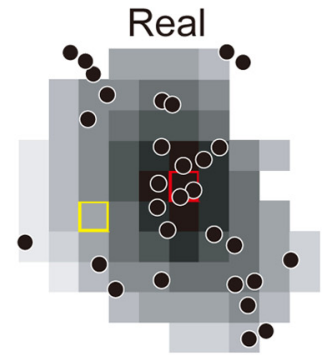

No. of particles $=30$

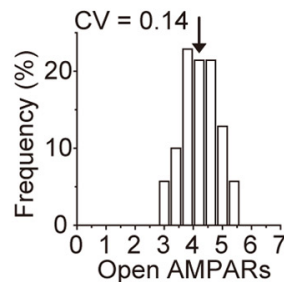

$\mathrm{F}$
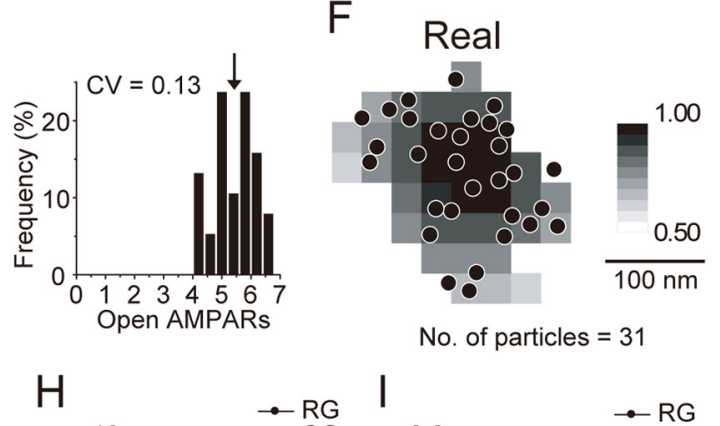

No. of particles $=31$

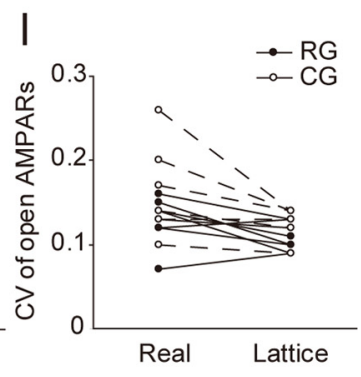

Figure 5. Small effects of uneven distribution of AMPARs on the AMPAR-mediated synaptic response. $A$, A replica image of a CG synapse. Scale bar, $100 \mathrm{~nm}$. The red line and the black dots indicate the edge of the synapse and the location of AMPAR immunoparticles, respectively. Gray squares were evenly placed at an interval of $30 \mathrm{~nm}$ within the synaptic area and indicate the locations of release sites tested with our simulations. Red (an "ON cluster" site) and yellow (an "OFF cluster" site) squares are the representative release sites and the simulation results from these sites are shown in $\boldsymbol{B}-\boldsymbol{D}$. $\boldsymbol{B}$, Glutamate concentration transients at each AMPAR location caused by a single quantal release of glutamate $(N=2000)$ at "ON cluster" (red) or "OFF cluster" (yellow) release sites were calculated and are shown in the upper traces (diffusion coefficient $=0.3 \mu \mathrm{m}^{2} / \mathrm{ms}$ ). Po of each AMPAR in response to the glutamate transient was calculated using the AMPAR kinetic model (Wadiche and Jahr, 2001) and shown in the lower traces. C, Summation of the Po of all AMPARs represents the cell's total response to the release of a single vesicle. Each trace in C shows the time course of the total open AMPAR number in response to release of glutamate at locations indicated in $A$. Red and yellow traces are responses from "ON cluster" and "OFF cluster" release, respectively. Distributions of the peak open AMPAR number are shown as histograms in $\boldsymbol{C}$ (bin width $=0.4$ open AMPARs). $\boldsymbol{D}$, Spatial distribution of the peak open AMPAR number in response to release from each location is expressed in gray scale. The location of release causing maximum response is shown as black $(=1)$ and responses from all other locations were scaled accordingly. The releases close to clusters of receptors (black dots) caused larger responses than releases in open spaces; however, the maximum difference was less than twofold. $\boldsymbol{E}$, Time course of the open AMPAR number in response to the release from various locations in a representative $R G$ synapse. The RG synapse, which has higher AMPAR density than the $C G$ synapses, showed a slightly higher number of the open AMPARs than the CG synapse with the similar number of immunoparticles as in $\boldsymbol{C}$. $\boldsymbol{F}$, Spatial distribution of the peak open AMPAR number for the RG synapse example. $\boldsymbol{G}$, Distribution of the peak open AMPAR number in real and lattice distribution in a synapse shown in $\boldsymbol{A}$. Inset is spatial distribution of the peak open AMPAR number in lattice distribution. The number of immunoparticles is $30 . \boldsymbol{H}$, Comparison of the average of open AMPARs between real and lattice distribution. There is no significant difference between them. I, Comparison of the CV of open AMPARs between real and lattice distribution. CV of real distribution was significantly higher than lattice distribution only in CG synapses. variability of synaptic response caused by release location (Fig. $5 H, I$ ).

In addition, spatial distribution of the peak open AMPAR number indicates that open AMPAR number in response to release at the center of the synapse is the largest and the response becomes smaller when the release occurs toward the periphery of the synapse (Fig. 5G). This tendency is also observed in the real (clustered) distribution (Figs. 5D, F; see also supplemental Fig. 5, available at www.jneurosci.org as supplemental material), indicating that not only the release location relative to the microclusters but also the release location relative to the center of the synapse would affect the amplitude of the AMPAR-mediated synaptic response. A three-dimensional diffusion model which takes into account of the multiple escape routes of glutamate from the cleft edge would likely emphasize this center-effect, as has been suggested by Franks et al. (2003).

The number of the receptors is the main factor in determining the amplitude of the AMPAR response

Simulation results conducted on all synapses from rat $\# 4$ is summarized in Figure 6. The average number of open AMPARs at the peak amplitude showed a strong positive correlation with the number of AMPAR immunoparticles in both types of synapses (Fig. 6A). This result is in accordance with the experiments showing the positive correlation of the AMPAR response with the spine size using two-photon glutamate uncaging in CA1 synapses (Matsuzaki et al., 2001) and with the theoretical predictions (Franks et al., 2002). We also found that the slopes of the regression lines between the open AMPAR number and the number of AMPAR immunoparticles were identical in both types of synapses, while the confidential intervals of their regression lines did not overlap between RG and CG synapses (regression line: $y=0.12 x+1.81$ in the RG synapses and $y=0.12 x+0.40$ in the CG synapses). This indicates that, for a given number of AMPAR immunoparticles, the average response size of the RG synapses is significantly higher than that of the CG synapse, and this is likely due to the higher AMPAR density in RG synapses. The overall average of AMPAR responses across all synapses, however, did not differ significantly between RG and CG types due to the large overlap of AMPAR numbers (average open AMPARs $=5.9 \pm 2.20$ for RG, $4.3 \pm$ 2.44 for CG, $p=0.108$, Student's $t$ test). Together, these results suggest that the average amplitude of the AMPAR response is deter- 
mined largely by the number of AMPARs expressed at individual synapses and that the synaptic area and the density of AMPARs have a minor role on the amplitude of AMPAR response within the size range $\left(<0.12 \mu \mathrm{m}^{2}\right)$ investigated in the present study.

The variability of open AMPAR numbers in response to various release locations in CG synapses was significantly higher than that in $\mathrm{RG}$ synapses $(\mathrm{CV}=$ $0.13 \pm 0.03$ in $\mathrm{RG}, \mathrm{CV}=0.17 \pm 0.05$ in CG, $p=0.010$, Student's $t$ test) with no correlation to the number of AMPAR immunoparticles (Fig. 6B, see also supplemental Fig. 5, available at www.jneurosci. org as supplemental material). Both CVs were similarly small across a wide range of values assigned for the number of glutamate within a single vesicle and the diffusion coefficient of glutamate in the extracellular space (supplemental Fig. 4, available at www.jneurosci.org as supplemental material). To understand how large the variability induced by release location difference compared with the overall variability in quantal response is, RG and CG fibers were stimulated in the presence of $\mathrm{Sr}^{2+}$ to desynchronize release and AMPAR-mediated quantal responses from each fiber were recorded electrophysiologically (supplemental Fig. 6, available at www.jneurosci.org as supplemental material). CV values of the peak amplitude of the quantal response from both fibers were similar (RG, $0.44 \pm 0.07$; CG, $0.45 \pm 0.13 ; n=3$ each), which were much larger than the $\mathrm{CV}$ values obtained by the above simulations. It is likely that the electrophysiologically recorded variation is largely due to the intersynaptic variability in quantal response as the $\mathrm{CV}$ value matches well with the value calculated for the number of immunogold at each synaptic contact $(0.48-0.84$; see supplemental Table 2, available at www.jneurosci.org as supplemental material). Comparisons of the other sources contributing to the intersynaptic and intrasynaptic quantal response variability are discussed in detail in supplemental discussion and in supplemental Figure 7, available at www.jneurosci.org as supplemental material. In short, we conclude that the quantal response variability introduced by the release location differences is very minor.

We also examined the influence of synaptic area and AMPAR density on the kinetics of the simulated AMPAR responses. The average rise and decay times of AMPAR responses had significant correlation with the synaptic area (Fig. 6C,D). The kinetics were faster for responses produced at synapses with a smaller synaptic area mainly due to the decreased presence of AMPARs experiencing lower concentration of glutamate which would have slower rise times. In addition, the delayed peaks of the responses from the low glutamate experiencing receptors would summate and contribute in producing slightly slower decay times as a whole. As the synaptic area of the RG synapses was half that of the CG synapses on average, a significant difference in kinetics was found between the two synapses (rise time: $0.18 \pm 0.021 \mathrm{~ms}$ in RG, $0.22 \pm 0.024 \mathrm{~ms}$ in CG, $p=0.003$; decay time: $1.47 \pm 0.044 \mathrm{~ms}$ in $\mathrm{RG}, 1.54 \pm 0.047 \mathrm{~ms}$ in CG, $p=0.003$, Mann-Whitney $U$ test). Faster kinetics of the AMPAR responses at RG synapses would be beneficial for reliably transmitting a high-frequency visual sensory signal from the retina. However, the effect of accelerating the synaptic transmission by making individual synaptic contacts compact appeared small and we could not detect any acceleration of the electrophysiologically recorded quantal response originating from RG synapse compared with those from CG synapse, although the recorded quantal responses were a collection of responses originating from multiple synaptic contacts, and the kinetics were probably affected by cable filtering, making it difficult to extract such small differences even if it existed (supplemental Fig. 6, available at www.jneurosci.org as supplemental material).

\section{The differential expression levels of NMDARs between RG and CG synapses and their effect on NMDAR-mediated quantal response evaluated by simulation}

In addition to AMPARs, NMDARs are also expressed at RG and CG synapses and are known to play key roles in synaptic transmission. To examine the quantitative and two-dimensional distribution of NMDARs and compare the difference in the expression pattern of the two kinds of ionotropic glutamate receptors between RG and CG synapses, we labeled the replicas obtained from 3 rats with an antibody for the NR1 subunit, which is an essential subunit for NMDARs to be functional (Moriyoshi et al., 1991; Forrest et al. 1994). RG synapses were identified with vGluT2 labeling as described above, and RG-relay cell synapses were distinguished from RG-IN synapses by M2 labeling (Fig. 7; supplemental Fig. 2, available at www.jneurosci.org as supplemental material).

Unlike AMPAR labeling, the immunoparticles for NR1 were distributed all over the IMP cluster in both RG and CG synapses (Figs. $7 A, B$ ). The localization of NR1 labeling did not coincide with the clearly visible IMPs. NR1 labeling was not particularly concentrated either in the center or in the periphery of the synaptic area. Similar to AMPAR labeling, the number of immunoparticles for NR1 showed a positive correlation with the synaptic area in both types of synapses (Fig. 7C; data from rat \#1 and supplemental Table 4, available at www.jneurosci.org as supplemental material). The mean number of immunoparticles for NR1 in the CG 

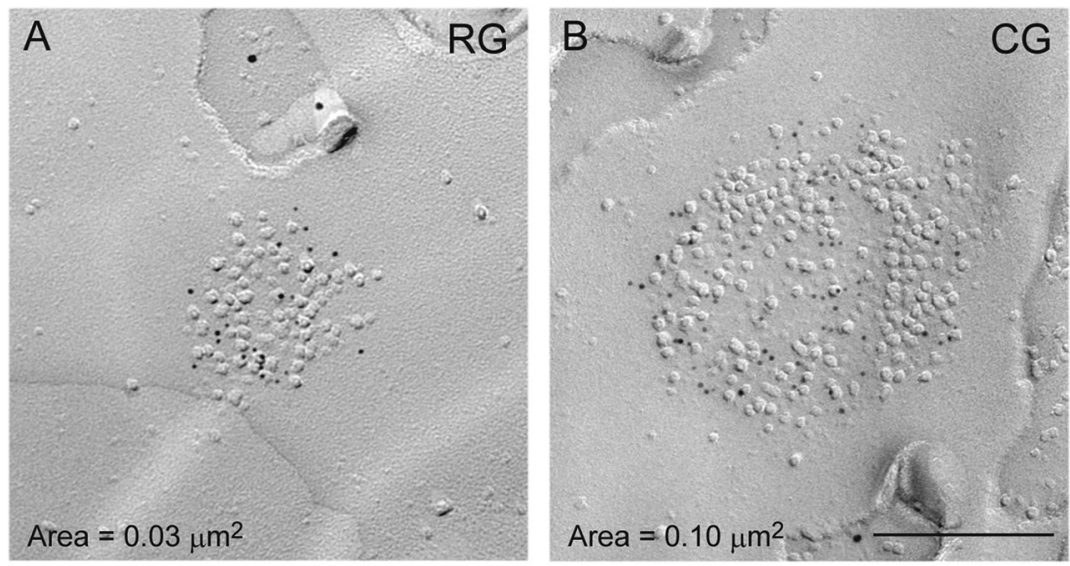

C
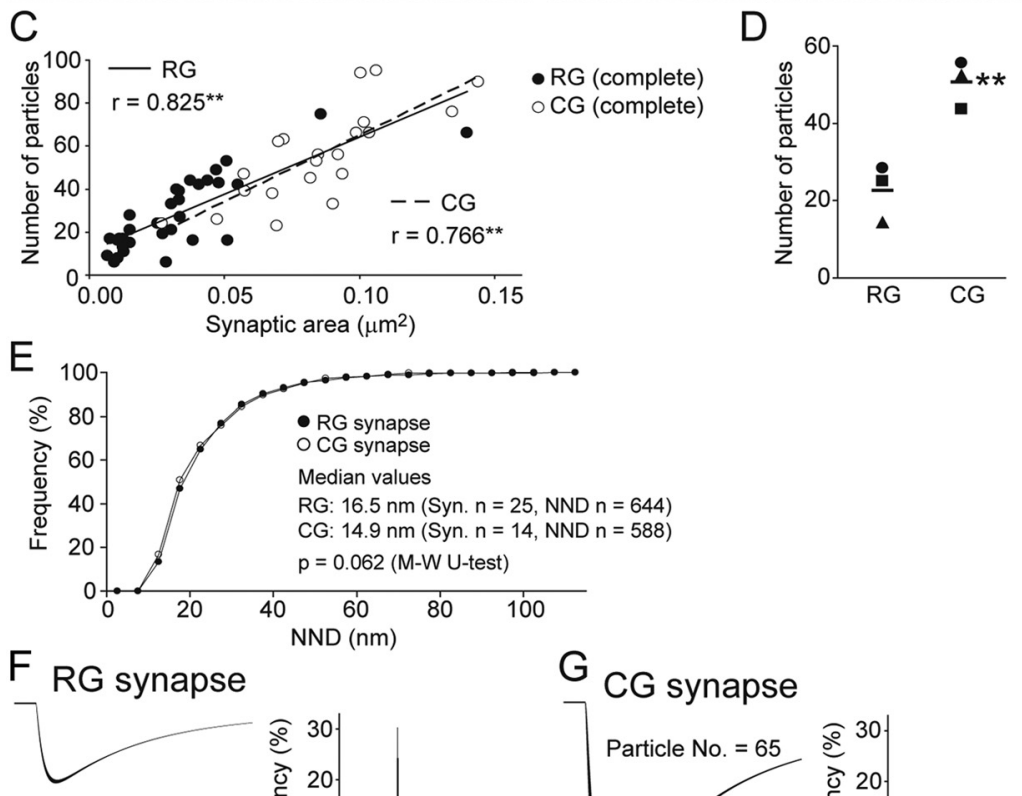

Particle No. $=22$

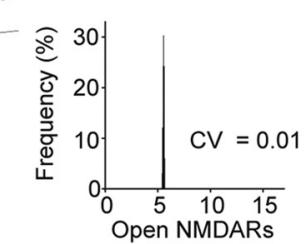

$\mathrm{H}$
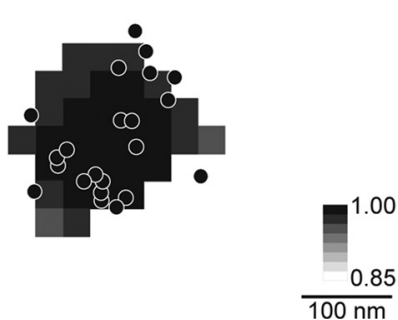

Figure 7. NMDARs are distributed similarly in RG and CG synapses and the amplitude of NMDAR-mediated synaptic response is determined by the number of receptors. $\boldsymbol{A}, \boldsymbol{B}$, Replica images of NR1 labeling in RG and CG synapses. Immunoparticles for NR1 (5 nm) accumulated over the IMP cluster areas of both types of synapses. Scale bar, $200 \mathrm{~nm}$. C, D, Quantitative analysis of NR1 labeling. C, Data from complete IMP clusters in rat \#1 are plotted. A positive correlation was found between the synaptic area and the number of NR1 immunoparticles in RG (filled symbols) and CG synapses (open symbols) ( $r=$ coefficient of correlation, ${ }^{* *} p<0.01$, Spearman's rank-order test). $\boldsymbol{D}$, The mean number of immunoparticles for NR1 in CG synapses was significantly higher than that in RG synapses in all three rats along with larger synaptic area of CG synapses (same symbols for individual rats, ${ }^{* *} p=0.007$, Student's $t$ test). Bars indicate mean values of three rats. $\boldsymbol{E}$, The distributions of NNDs of NR1 immunoparticles in RG and CG synapses were virtually the same. $\boldsymbol{F}-\boldsymbol{I}$, Glutamate diffusion was calculated as in Figure $5(N=2000, D=0.3)$, and a modified NMDAR kineticmodel (Lester and Jahr, 1992) (see Materials and Methods) was run for each NMDAR, the location of which was measured in $\boldsymbol{A}$ and $\boldsymbol{B}$. Subsequently, the summation of all Pos of NMDARs in response to release at each location within the synapse was calculated. $\boldsymbol{F}$, Thirty three traces of the open NMDAR time course, which corresponds to NMDAR-mediated response to 33 release locations tested, are overlaid for a RG synapse example with 22 particles. Very little dependence of NMDAR response on release location was found as apparent from the histogram (bin width $=0.05$ and 0.25 open NMDARs for $\boldsymbol{F}$ and $\boldsymbol{G}$, respectively). G, One-hundred and fourteen traces are overlaid for a CG synapse example with 65 particles synapses was 2 times higher than that in the RG synapses in all 3 rats (Fig. 7D; $24.3 \pm$ 4.71 in the RG synapses and $49.8 \pm 7.54$ in the CG synapses, $p=0.007$, Student's $t$ test). When the local distribution of the NR1 immunoparticles was compared between the two synapses by NND measurement, the two distributions overlapped almost completely (Fig. $7 E$; data from rat \#2), indicating an identical clustering pattern for NMDARs in the two types of synapses.

Since NMDARs have a higher affinity for glutamate, it appears likely that NMDARmediated synaptic responses would be even less variable regardless of the location of the synaptic vesicular release compared with the AMPAR-mediated responses. To confirm this notion, we simulated the NMDAR response by running the kinetic model of NMDARs (Lester and Jahr, 1992) using the same glutamate diffusion model as described above and the location of NMDARs measured from representative replica images of each type of synapse (Fig. 7F, G). As expected, the variability of the NMDAR response caused by different release sites within synaptic contact was $\sim 13$ times smaller than that of the AMPAR response (Fig. 7F-I; supplemental Movie 2, available at www.jneurosci.org as supplemental material), which suggests that NMDARs can stably respond to glutamate release regardless of the release site. In addition, the amplitude of the NMDAR-mediated responses in the two synapses having approximately a threefold difference in the number of immunoparticles resulted in approximately a threefold difference (Fig. $7 F, G$ ), suggesting that the number of NMDARs is the dominant determinant of NMDAR-mediated responses.

\section{NMDAR-mediated response is larger in the CG synapses than in the RG synapses}

Our SDS-FRL analysis revealed higher expression level of the NMDARs in CG synapses with similar AMPAR expression level between CG and RG synapses, and our simulation demonstrated the number of receptors as a primary determinant of the amplitude of the synaptic response. Therefore, to confirm the physiological relevance of the anatomical and simula-

\section{$\leftarrow$}

$\boldsymbol{H}, \boldsymbol{I}$, Spatial maps of the number of peak open NMDARs in response to the vesicular release at each location within the synaptic area. The release location giving the maximal NMDAR response in each synapse is shown in black $(=1)$ and responses from all other release locations are scaled accordingly (range $0.85-1)$. Note that the peak NMDAR responses were nearly independent from both the receptor distributions and the release locations. 
tion data presented here, we performed whole-cell voltage-clamp recordings from the relay cells in acute slice preparations and recorded the evoked EPSCs by stimulating RG and CG fibers, respectively. EPSCs from RG and CG fiber stimulation could readily be distinguished with the former having paired-pulse depression and the latter having paired-pulse facilitation (Fig. 8A,B) (Turner and Salt, 1998). The evoked EPSCs were recorded at holding potentials of $-77 \mathrm{mV}$ and $+33 \mathrm{mV}$ to evaluate the relative amount of AMPAR and NMDAR activation by synaptic glutamate release, respectively (Fig. $8 \mathrm{C}$ ). As expected from the morphological observations and the simulation studies shown above, stimulation of the CG fiber caused larger NMDAR activation relative to AMPAR activation compared with the RG fiber stimulation (Figs. 8C,D) (NMDA/ AMPA ratio calculated at the above potentials; RG, $0.53 \pm 0.30, n=8$ cells; CG, $2.63 \pm 0.62, n=5$ cells). These data suggest that the distinct relative expression levels of synaptic NMDAR and AMPARs underlie the distinct NMDA/AMPA response ratios in RG and CG synapses.

\section{Discussion}

Using SDS-FRL, we have identified large diversity in the expression level and arrangement of synaptic glutamate receptors in the dLGN neurons. Using simple simulations, large diversity in synaptic responses across synapses (i.e., intersynaptic variability) was estimated as well. However, the variability of synaptic responses within single synapses (i.e., intrasynaptic variability) was estimated to be only slightly affected by the inhomogeneous two-dimensional distribution of receptors. We conclude that the receptor number predominantly determines the average amplitude of quantal responses at each synapse, and that the postsynaptic profiles of synapses may be formed under a structural constraint to keep this simple rule.

\section{Physiological implications of distinct intrasynaptic arrangements of glutamate receptors}

RG and CG fibers are classified as the driver and modulator, respectively (Sherman and Guillery, 1998). An action potential firing of a single RG fiber triggers vesicle release at many synapses formed on a single postsynaptic relay cell (Paulsen and Heggelund, 1994) and as a consequence the relay cell often discharges. Such reliable synapse would allow faithful conveyance of information from the retina to the cortex.

In accordance with previous experimental and theoretical studies (Matsuzaki et al., 2001; Franks et al., 2002), our simulation analysis suggest that the synaptic strength is primarily determined by the number of AMPARs within a synapse. We anticipated that RG synapses would accomplish its reliable transmission in part by having large number of AMPARs. However, the number of AMPAR expressed in individual RG synapses was found to be the same as that in the CG synapses. Instead, we found that the synaptic area of the RG synapses was smaller than that of the CG synapses resulting in a high AMPAR density among the RG synapses. Our simulations showed that higher AMPAR density would increase the amplitude of AMPAR responses, and the compact RG synaptic area would accelerate the rise and decay times of the AMPAR responses. Whether this $\sim 1.5$ open AMPAR enhancement of the peak quantal response amplitude by the smaller RG synapse size (Fig. $6 \mathrm{~A}$ ) contributes to fast and reliable RG synaptic transmission or not remains to be further elucidated. As one RG fiber makes multiple synapses on a single relay cell [on average 17 synapses according to Robson (1993)], depending on the release probability, the small increment could add up on average. However, since the variability of the number of AMPAR expressed on individual synapse is large and other sources of variability (see supplemental discussions) would also come into play, careful consideration of each factor is required to evaluate whether the benefit of the smaller synapse size survives through the variability and is significant in the total synaptic response.

In contrast to the RG fiber, a single CG fiber forms only $1-2$ synapses onto individual cells, resulting in a small synaptic response, and large variability of synaptic strength among individual CG synapses is generated by different numbers of receptors expressed at individual synapses. We found that the number of NMDARs expressed in individual synapses was twice in the CG compared with $R G$ synapses due to their larger synaptic area. We also found that the response at the CG synapses showed higher NMDA/AMPA ratio than that at the RG synapses. It has been reported that the augmentation of synaptic responses was observed selectively in CG synapse in a frequency-dependent manner by summation of NMDAR- 
mediated current (Miyata and Imoto, 2006). Thus, higher expression level of NMDARs in CG synapses may contribute to the temporal summation of CG responses, and both the larger synaptic area and the inhomogeneous distribution of AMPARs found in CG synapses may be required to accommodate larger number of NMDARs.

It should be noted that $\sim 4.9$ times higher NMDA/AMPA ratio was measured in the CG synapses compared with RG synapses by our electrophysiological recordings, which was higher than the twofold difference found by the NR1 immunolabeling at individual synapses. Bundles of CG fibers, most of which did not form direct synaptic contacts with the recorded cell, were likely to be stimulated in our electrophysiological recordings. The above difference could be explained if CG synapses were more prone to glutamate spillover. Closer inspection of the structure and the molecule distributions would be required to draw further conclusion. Another possibility is the developmental difference in the NMDA/AMPA ratio, which would also be of interest to pursue further.

\section{Intrasynaptic microclusters of AMPARs}

In this study, we revealed differential intrasynaptic arrangement of AMPARs in physiologically distinct RG and CG synapses. Individual CG synapses express similar number of AMPARs in a larger synaptic area than RG synapses and microclusters of AMPARs were detected in both synapses, which result in larger intrasynaptic areas devoid of AMPAR expression in CG synapses than those in RG synapses. In a previous study, we showed inputspecific patterns of AMPAR distribution and microclusters in CF-PC and PF-PC synapses (Masugi-Tokita et al., 2007). A study using single-molecule imaging has also predicted anchored AMPARs forming microclusters of AMPARs within individual hippocampal synapses (Heine et al., 2008). Therefore, microclusters of AMPARs appear to be a common representation of AMPARs in the postsynaptic membrane specialization.

\section{The effect of release location on quantal postsynaptic response}

Ultrastructural and biochemical studies have revealed the presence of a regularly arranged meshwork of protein complexes over the presynaptic active zone, which consist of vesicle release related proteins, cytoskeletal proteins, and cell adhesion molecules (Phillips et al., 2001). Docked vesicles have also been observed in multiple parts of single active zones, suggesting various release locations within a single presynaptic bouton (Schikorski and Stevens, 1997). We have shown that AMPARs distributed unevenly in CG synapses, and AMPAR responses to each quantal release could be different depending on the release location (Fig. 5). Quantal release in close proximity to microclusters would likely produce a larger response than elsewhere. However, the release location relative to the center of the synapse also has a large contribution to the response size as demonstrated by the lattice distribution simulations. It appears possible to increase the efficacy and stability of synaptic transmission by locking the release site in proximity to the microclusters or the center of the synapse, and synaptic plasticity could be realized by just such coordination. Thus, detecting the location of AMPAR microclusters relative to release sites would be an important issue to be further elucidated in future analysis.

\section{The amplitude of AMPAR and NMDAR quantal response primarily depends on the number of receptors expressed} If the dimension of the synapse is such that the released glutamate can cover the entire synaptic area with sufficiently high concentration relative to the affinity of the receptors, then the synaptic strength would likely be determined by the total number of the receptors. On the other hand, if the actions of the glutamate remain local, the local density of the receptors would primarily dictate the synaptic strength. Our simulations based on actual geometrical information of receptor distribution showed that, although the local density of AMPARs has an influence on the amplitude of each quantal event, it has a minor effect on the average size of the synaptic AMPAR response (Figs. 5, 6). Our results indicate that the average AMPAR response is primarily proportional to the number of AMPARs in individual synapses (Fig. 6A), and thus, where the number of AMPARs is proportional to the synaptic area (Fig. 3A) (Takumi et al., 1999; Tanaka et al., 2005), the strength of the synapse can be approximately estimated by the size of the synapse. Extreme profiles to which this simple rule does not apply have never been encountered. One caveat is that this simple estimation could only be applied to the comparison of the synaptic strength within a single synapse subtype formed in a single cell type, as synapses receiving different input sources would have different average receptor densities as demonstrated by our comparison between the RG and CG synapses. Thus, a pair of RG and CG synapses with almost identical synapse sizes would be expected to have different synaptic strength.

NMDAR responses at single synapses showed even higher stability than that of AMPAR responses regardless of release locations. Higher affinity for glutamate of NMDARs (EC ${ }_{50}$ values, 500-560 $\mu \mathrm{M}$ in AMPARs, $0.4-1.8 \mu \mathrm{M}$ in NMDARs; Dingledine et al., 1999) enables this stable response, and all NMDARs in individual synapses would have a similar chance of being activated despite the uneven distribution of NMDARs. This high affinity of NMDARs would also make its responses impervious to the density of the receptors and only the number of the receptors in the synapse governs the response.

In conclusion, we revealed the input-specific and receptor-typespecific intrasynaptic arrangements of AMPARs and NMDARs in the RG and CG synapses in the dLGN relay cells. We found that the number of the receptors is a major determinant factor of the amplitude of their postsynaptic quantal responses, and that the synaptic area and the arrangement of the ionotropic glutamate receptors have a minor influence on the amplitude, the kinetics, and the distinct properties of synaptic transmission at single synapses.

\section{References}

Barbour B (2001) An evaluation of synapse independence. J Neurosci 21:7969-7984

Baude A, Nusser Z, Molnár E, Mcllhinney RA, Somogyi P (1995) Highresolution immunogold localization of AMPA type glutamate receptor subunits at synaptic and non-synaptic sites in rat hippocampus. Neuroscience 69:1031-1055.

Chen N, Ren J, Raymond LA, Murphy TH (2001) Changes in agonist concentration dependence that are a function of duration of exposure suggest $\mathrm{N}$-methyl-D-aspartate receptor nonsaturation during synaptic stimulation. Mol Pharmacol 59:212-219.

Dingledine R, Borges K, Bowie D, Traynelis SF (1999) The glutamate receptor ion channels. Pharmacol Rev 51:7-61.

Erişir A, Van Horn SC, Bickford ME, Sherman SM (1997) Immunocytochemistry and distribution of parabrachial terminals in the lateral geniculate nucleus of the cat: a comparison with corticogeniculate terminals. J Comp Neurol 377:535-549.

Forrest D Yuzaki M, Soares HD, Ng L, Luk DC, Sheng M, Stewart CL, Morgan JI, Connor JA, Curran T (1994) Targeted disruption of NMDA receptor 1 gene abolishes NMDA response and results in neonatal death. Neuron 13:325-338.

Franks KM, Bartol TM Jr, Sejnowski TJ Jr (2002) A Monte Carlo model reveals independent signaling at central glutamatergic synapses. Biophys J 83:2333-2348.

Franks KM, Stevens CF, Sejnowski TJ (2003) Independent sources of quantal variability at single glutamatergic synapses. J Neurosci 23:3186-3195.

Fremeau RT Jr, Troyer MD, Pahner I, Nygaard GO, Tran CH, Reimer RJ, 
Bellocchio EE, Fortin D, Storm-Mathisen J, Edwards RH (2001) The expression of vesicular glutamate transporters defines two classes of excitatory synapse. Neuron 31:247-260.

Fujimoto K (1995) Freeze-fracture replica electron microscopy combined with SDS digestion for cytochemical labeling of integral membrane proteins. Application to the immunogold labeling of intercellular junctional complexes. J Cell Sci 108:3443-3449.

Fujiyama F, Hioki H, Tomioka R, Taki K, Tamamaki N, Nomura S, Okamoto K, Kaneko T (2003) Changes of immunocytochemical localization of vesicular glutamate transporters in the rat visual system after the retinofugal denervation. J Comp Neurol 465:234-249.

Hagiwara A, Fukazawa Y, Deguchi-Tawarada M, Ohtsuka T, Shigemoto R (2005) Differential distribution of release-related proteins in the hippocampal CA3 area as revealed by freeze-fracture replica labeling. J Comp Neurol 489:195-216.

Harris KM, Landis DM (1986) Membrane structure at synaptic junctions in area CA1 of the rat hippocampus. Neuroscience 19:857-872.

Heine M, Groc L, Frischknecht R, Béique JC, Lounis B, Rumbaugh G, Huganir RL, Cognet L, Choquet D (2008) Surface mobility of postsynaptic AMPARs tunes synaptic transmission. Science 320:201-205.

Iwasato T, Datwani A, Wolf AM, Nishiyama H, Taguchi Y, Tonegawa S, Knöpfel T, Erzurumlu RS, Itohara S (2000) Cortex-restricted disruption of NMDAR1 impairs neuronal patterns in the barrel cortex. Nature 406:726-731.

Kharazia VN, Weinberg RJ (1997) Tangential synaptic distribution of NMDA and AMPA receptors in rat neocortex. Neurosci Lett 238:41-44.

Kulik A, Nakadate K, Nyíri G, Notomi T, Malitschek B, Bettler B, Shigemoto R (2002) Distinct localization of GABA(B) receptors relative to synaptic sites in the rat cerebellum and ventrobasal thalamus. Eur J Neurosci 15:291-307.

Lester RA, Jahr CE (1992) NMDA channel behavior depends on agonist affinity. J Neurosci 12:635-643.

Li J, Wang S, Bickford ME (2003) Comparison of the ultrastructure of cortical and retinal terminals in the rat dorsal lateral geniculate and lateral posterior nuclei. J Comp Neurol 460:394-409.

Lieberman AR, Webster KE (1974) Aspects of the synaptic organization of intrinsic neurons in the dorsal lateral geniculate nucleus. An ultrastructural study of the normal and of the experimentally deafferented nucleus in the rat. J Neurocytol 3:677-710.

Masugi-Tokita M, Tarusawa E, Watanabe M, Molnár E, Fujimoto K, Shigemoto R (2007) Number and density of AMPA receptors in individual synapses in the rat cerebellum as revealed by SDS-digested freeze-fracture replica labeling. J Neurosci 27:2135-2144.

Matsubara A, Laake JH, Davanger S, Usami S, Ottersen OP (1996) Organization of AMPA receptor subunits at a glutamate synapse: a quantitative immunogold analysis of hair cell synapses in the rat organ of Corti. J Neurosci 16:4457-4467.

Matsui K, Jahr CE, Rubio ME (2005) High-concentration rapid transients of glutamate mediate neural-glial communication via ectopic release. J Neurosci 25:7538-7547.

Matsui M, Motomura D, Fujikawa T, Jiang J, Takahashi S, Manabe T, Taketo MM (2002) Mice lacking M2 and M3 muscarinic acetylcholine receptors are devoid of cholinergic smooth muscle contractions but still viable. J Neurosci 22:10627-10632.

Matsuzaki M, Ellis-Davies GC, Nemoto T, Miyashita Y, Iino M, Kasai H (2001) Dendritic spine geometry is critical for AMPA receptor expression in hippocampal CA1 pyramidal neurons. Nat Neurosci 4:1086-1092.

McLarnon JG, Curry K (1990) Single channel properties of the N-methylD-aspartate receptor channel using NMDA and NMDA agonists: on-cell recordings. Exp Brain Res 82:82-88.

Miyata M, Imoto K (2006) Different composition of glutamate receptors in corticothalamic and lemniscal synaptic responses and their roles in the firing responses of ventrobasal thalamic neurons in juvenile mice. J Physiol 575:161-174.

Miyazaki T, Fukaya M, Shimizu H, Watanabe M (2003) Subtype switching of vesicular glutamate transporters at parallel fibre-Purkinje cell synapses in developing mouse cerebellum. Eur J Neurosci 17:2563-2572.

Moriyoshi K, Masu M, Ishii T, Shigemoto R, Mizuno N, Nakanishi S (1991) Molecular cloning and characterization of the rat NMDA receptor. Nature 354:31-37.

Murphy PC, Sillito AM (1987) Corticofugal feedback influences the generation of length tuning in the visual pathway. Nature 329:727-729.

Nicholson DA, Geinisman Y (2009) Axospinous synaptic subtype-specific differences in structure, size, ionotropic receptor expression, and connectivity in apical dendritic regions of rat hippocampal CAl pyramidal neurons. J Comp Neurol 512:399-418.
Nielsen TA, DiGregorio DA, Silver RA (2004) Modulation of glutamate mobility reveals the mechanism underlying slow-rising AMPAR EPSCs and the diffusion coefficient in the synaptic cleft. Neuron 42:757-771.

Nusser Z, Lujan R, Laube G, Roberts JD, Molnar E, Somogyi P (1998) Cell type and pathway dependence of synaptic AMPA receptor number and variability in the hippocampus. Neuron 21:545-559.

Nyíri G, Stephenson FA, Freund TF, Somogyi P (2003) Large variability in synaptic $\mathrm{N}$-methyl-D-aspartate receptor density on interneurons and a comparison with pyramidal-cell spines in the rat hippocampus. Neuroscience 119:347-363.

Paulsen O, Heggelund P (1994) The quantal size at retinogeniculate synapses determined from spontaneous and evoked EPSCs in guinea-pig thalamic slices. J Physiol 480:505-511.

Peters A, Palay SL, Webster HDF (1991) The fine structure of the nervous system. New York: Oxford UP.

Phillips GR, Huang JK, Wang Y, Tanaka H, Shapiro L, Zhang W, Shan WS, Arndt K, Frank M, Gordon RE, Gawinowicz MA, Zhao Y, Colman DR (2001) The presynaptic particle web: ultrastructure, composition, dissolution, and reconstitution. Neuron 32:63-77.

Plummer KL, Manning KA, Levey AI, Rees HD, Uhlrich DJ (1999) Muscarinic receptor subtypes in the lateral geniculate nucleus: a light and electron microscopic analysis. J Comp Neurol 404:408-425.

Rafols JA, Valverde F (1973) The structure of the dorsal lateral geniculate nucleus in the mouse. A Golgi and electron microscopic study.J Comp Neurol 150:303-332.

Reese BE (1984) The projection from the superior colliculus to the dorsal lateral geniculate nucleus in the rat. Brain Res 305:162-168.

Robson JA (1993) Qualitative and quantitative analysis of the patterns of retinal input to neurons in the dorsal lateral geniculate nucleus of the cat. J Comp Neurol 334:324-336.

Rollenhagen A, Lübke JH (2006) The morphology of excitatory central synapses: from structure to function. Cell Tissue Res 326:221-237.

Rusakov DA (2001) The role of perisynaptic glial sheaths in glutamate spillover and extracellular $\mathrm{Ca}(2+)$ depletion. Biophys J 81:1947-1959.

Sandri C, Akert K, Livingston RB, Moor H (1972) Particle aggregations at specialized sites in freeze-etched postsynaptic membranes. Brain Res 41:1-16.

Savtchenko LP, Rusakov DA (2007) The optimal height of the synaptic cleft. Proc Natl Acad Sci U S A 104:1823-1828.

Schikorski T, Stevens CF (1997) Quantitative ultrastructural analysis of hippocampal excitatory synapses. J Neurosci 17:5858-5867.

Sherman SM, Guillery RW (1998) On the actions that one nerve cell can have on another: distinguishing "drivers" from "modulators". Proc Natl Acad Sci U S A 95:7121-7126.

Sillito AM, Cudeiro J, Murphy PC (1993) Orientation sensitive elements in the corticofugal influence on centre-surround interactions in the dorsal lateral geniculate nucleus. Exp Brain Res 93:6-16.

Takumi Y, Ramírez-León V, Laake P, Rinvik E, Ottersen OP (1999) Different modes of expression of AMPA and NMDA receptors in hippocampal synapses. Nat Neurosci 2:618-624.

Tanaka J, Matsuzaki M, Tarusawa E, Momiyama A, Molnar E, Kasai H, Shigemoto R (2005) Number and density of AMPA receptors in single synapses in immature cerebellum. J Neurosci 25:799-807.

Turner JP, Salt TE (1998) Characterization of sensory and corticothalamic excitatory inputs to rat thalamocortical neurones in vitro. J Physiol 510:829-843.

Usrey WM, Reppas JB, Reid RC (1998) Paired-spike interactions and synaptic efficacy of retinal inputs to the thalamus. Nature 395:384-387.

Uteshev VV, Pennefather PS (1996) A mathematical description of miniature postsynaptic current generation at central nervous system synapses. Biophys J 71:1256-1266.

Van Horn SC, Erişir A, Sherman SM (2000) Relative distribution of synapses in the A-laminae of the lateral geniculate nucleus of the cat. J Comp Neurol 416:509-520.

Wadiche JI, Jahr CE (2001) Multivesicular release at climbing fiberPurkinje cell synapses. Neuron 32:301-313.

Xu-Friedman MA, Regehr WG (2004) Structural contributions to shortterm synaptic plasticity. Physiol Rev 84:69-85.

Zheng K, Scimemi A, Rusakov DA (2008) Receptor actions of synaptically released glutamate: the role of transporters on the scale from nanometers to microns. Biophys J 95:4584-4596.

Zuber B, Nikonenko I, Klauser P, Muller D, Dubochet J (2005) The mammalian central nervous synaptic cleft contains a high density of periodically organized complexes. Proc Natl Acad Sci U S A 102:19192-19197. 